\title{
Parity-violating deep-inelastic electron-deuteron scattering: Higher twist and parton angular momentum
}

\author{
Chien-Yeah Seng ${ }^{1}$ and Michael J. Ramsey-Musolf ${ }^{1,2}$ \\ ${ }^{1}$ University of Wisconsin-Madison, Madison, Wisconsin 53706, USA \\ ${ }^{2}$ California Institute of Technology, Pasadena, California 91125, USA \\ (Received 1 March 2013; revised manuscript received 13 June 2013; published 15 July 2013)
}

\begin{abstract}
We study the effect of parton angular momentum on the twist-four correction to the left-right asymmetry in the electron-deuteron parity-violating deep-inelastic scattering (PVDIS). We show that this higher-twist correction is transparent to the dynamics of parton angular momentum needed to account for the Sivers and Boer-Mulders functions and spin-independent parton distribution functions. A sufficiently precise measurement of the PVDIS asymmetry may, thus, provide additional information about the parton dynamics responsible for nucleon spin.
\end{abstract}

DOI: 10.1103/PhysRevC.88.015202

PACS number(s): 24.85.+p, 13.60.Hb, 11.80.-m, 11.10.St

\section{INTRODUCTION}

As a complement to the studies at high-energy frontier, measurements at the intensity frontier (or precision frontier) provide powerful tools in the search for physics beyond the standard model (BSM). Observables such as the muon anomalous magnetic moment are measured to very high precision, and experimental results are then compared with theoretical predictions. To the extent that the latter are sufficiently reliable, any possible deviation would point to BSM physics. Alternately, these experiments can provide new insights into the dynamics of the standard model.

Electron-deuteron parity-violating deep-inelastic scattering ( $e$ D PVDIS) is an excellent example of this class of studies. Historically, it provided the first experimental measurement of weak mixing angle $\theta_{W}$ [1]. Nowadays, with the prospect of the Jefferson Laboratory $12-\mathrm{GeV}$ upgrade and the use of a new spectrometer called SoLID, the left-right asymmetry of PVDIS can be measured with $0.5 \%$ precision over the kinematic range $0.3<x_{B}<0.7$ [2]. With this level of precision, one will be able to probe or constrain an interesting set of BSM scenarios, such as a leptophobic $Z^{\prime}$ boson [3,4] and supersymmetry [5], as well as to study hadronic physics effects, which are yet to be fully understood, such as charge symmetry violation (CVC) and higher-twist (HT).

The effect of HT [6] is a potentially important standard model correction that originates from the interaction between partons. This correction in general scales as $\left(Q^{2}\right)^{-(\tau-2) / 2}$, with the twist $\tau>2$, so its effect is enhanced at low $Q^{2}$. In the framework of the operator product expansion (OPE), the higher-twist correction can be expressed as a convolution of a high-energy and low-energy piece; the former (embodied in the Wilson coefficients) can be calculated using perturbative methods, whereas the latter involves hadronic matrix elements that require understanding of nonperturbative QCD. Studying the higher-twist correction may help us in probing correlations between the confined quarks and gluons inside the nucleon, so it is interesting to explore HT matrix elements within various model approaches. One advantage of $e$ D PVDIS process is that the HT contribution to the leading term in the PV asymmetry (defined below) arises from a single operator matrix element and can, in principle, be separated kinematically from the subleading terms that have a more complicated HT structure. With this motivation in mind, several previous works [7-10] have been carried out to study the twist-four (i.e., $\tau=4$ ) correction to the left-right asymmetry of $e \mathrm{D}$ PVDIS. In what follows, we report on a study that follows up these earlier works.

The study of HT may also shed light on another important issue, namely, the spin structure of the nucleon. Nearly twentyfive years ago, the EMC collaboration [11] performed a DIS experiment with longitudinally-polarized muons on a target of longitudinally polarized protons, obtaining a value for the structure function $g_{1}\left(x_{B}\right)$ over the range $0.01<x_{B}<0.7$. After extrapolating to the low- and high- $x_{B}$ region, the collaboration obtained a value for the leading moment of $g_{1}\left(x_{B}\right)$ that contradicted the Ellis-Jaffe sum rule [12] and implied that the spin of a proton is not built up entirely from the quark spin. The result has been confirmed by a variety of subsequent studies. A key question in nuclear physics research has thus become explaining in detail the source of nucleon spin in terms of QCD degrees of freedom.

From a theoretical perspective, arriving at a decomposition of the nucleon spin in terms of gauge-invariant matrix elements of local operators that afford a straightforward partonic interpretation has been a vexing problem, and different approaches have been pursued over the years [13-17]. In each case, reference is usually made to the interpretation in the light cone-gauge dependence notwithstanding — given its historical importance for thinking about parton dynamics. However, while the meaning of the quark helicity is gauge invariant, the relative importance of other aspects of partonic angular momentum (gluon helicity and quark and gluon orbital angular momentum) in general vary with the choice of gauge and even definition. Nonetheless, it is interesting to ask how different observables may probe different aspects of partonic angular momentum and to do so in a way that is both gauge invariant and as insensitive as possible to a particular angular momentum decomposition.

In this respect, we will study HT in the context of light-cone quantization. In early work within this framework, it has been shown that one particular component of parton angular momentum, identified as quark orbital angular momentum (OAM) 
under light-cone quantization using light-cone gauge, is responsible for the nonzero value of Sivers function and BoerMulders function [18,19] in semi-inclusive deep-inelastic scattering (SIDIS) [21]. In light of these results, it is also interesting to study how the inclusion of the same component of parton angular momentum modifies the current model predictions for HT corrections to $e \mathrm{D}$ PVDIS. Indeed, in all the previous studies of $e \mathrm{D}$ PVDIS, only the Fock component of the nucleon wave function with zero parton OAM has been included.

After including quark OAM in the light-cone amplitudes, we observe a rather nonintuitive phenomenon: although the absolute magnitude of individual nonzero quark OAM contributions can be significant, they largely cancel against each other. We will argue that this cancellation is largely independent of the detailed model for the relevant light-cone amplitudes. As a result, the twist-four correction to PVDIS is almost transparent to the inclusion of quark OAM. In contrast, other hadronic quantities, such as the parton distribution functions (PDFs), Sivers function, and Boer-Mulders function, manifest non-negligible dependence on quark OAM. Generalizing from the particular choice of light-cone quantization and light-cone gauge, we thus conclude that whatever features of parton angular momentum are responsible for the observed behavior of the PDFs, Sivers, and Boer-Mulders functions, they should have a relatively minor impact on the HT correction to $e$ D PVDIS of interest here. Moreover, any deviation from the light-cone predictions obtained here and in previous works [7-9], should they be observed experimentally, would signal the importance of other aspects of parton angular momentum and/or higher Fock space components of the nucleon wave function.

The discussion of the computation leading to these observations is arranged in the following order: in Sec. II we summarize the relevant results of the general formulation of the twist-four correction to $e$ D PVDIS; in Sec. III we introduce the light-cone wave function with quark OAM dependence; in Sec. IV we present the analytic expressions of the hadronic matrix elements needed for the twist-four correction, and demonstrate the generic cancellation between nonzero quark OAM components; in Sec. V we present the numerical results using one specific choice of nucleon wave function, and discuss their physical significance. Detailed formulas appear in the Appendix.

\section{HIGHER TWIST IN PVDIS: GENERAL FORMULATION}

Here, we review the well-known results for the twist-four correction in $e \mathrm{D}$ PVDIS. We will simply quote the central equations that are relevant to our study without any derivation and refer the reader to Refs. [8,9] for the details.

In $e \mathrm{D}$ PVDIS, longitudinally polarized electron beams are incident on unpolarized deuteron targets. One measures the PV right-left asymmetry

$$
A_{\mathrm{RL}}=\frac{d \sigma_{R}-d \sigma_{L}}{d \sigma_{R}+d \sigma_{L}}
$$

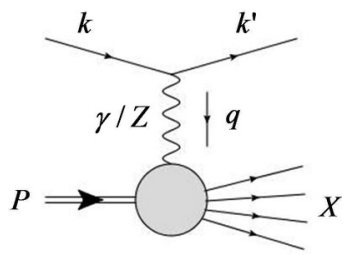

FIG. 1. Kinematics of e-D PVDIS: a deuteron of momentum $P$ interacts with an incoming electron of momentum $k$ via an exchange of a single photon or $z$ boson, and breaks into hadrons, which are denoted collectively as $X$.

where $d \sigma_{R / L}$ is the differential cross section for the scattering of the right-/left-handed electrons. At the one-boson exchange (OBE) level, the leading parity-violating piece comes from the interference between photon and $Z$-boson exchange diagrams (see Fig. 1). The low-energy $Z$-boson exchange interaction can be described by the following effective four-fermion interaction:

$$
\begin{aligned}
\mathcal{L}_{P V}= & \frac{G_{F}}{\sqrt{2}}\left[\bar{e} \gamma^{\mu} \gamma_{5} e\left(C_{1 u} \bar{u} \gamma_{\mu} u+C_{1 d} \bar{d} \gamma_{\mu} d\right)\right. \\
& \left.+\bar{e} \gamma^{\mu} e\left(C_{2 u} \bar{u} \gamma_{\mu} \gamma_{5} u+C_{2 d} \bar{d} \gamma_{\mu} \gamma_{5} d\right)\right],
\end{aligned}
$$

where, at tree level, we have

$$
\begin{aligned}
& C_{1 u}=-\frac{1}{2}+\frac{4}{3} \sin ^{2} \theta_{W}, \\
& C_{1 d}=\frac{1}{2}-\frac{2}{3} \sin ^{2} \theta_{W}, \\
& C_{2 u}=-\frac{1}{2}+2 \sin ^{2} \theta_{W}, \\
& C_{2 d}=\frac{1}{2}-2 \sin ^{2} \theta_{W} .
\end{aligned}
$$

Neglecting contributions from sea quarks, assuming charge symmetry $\left(u_{V}^{p}=d_{V}^{n}\right.$, etc. with $q_{V}^{N}$ being the valence quark PDF of nucleon $N$ ), the leading-twist SM prediction is given by the Cahn-Gilman formula [20]

$A_{\mathrm{RL}}=\frac{G_{F} Q^{2}}{2 \sqrt{2} \pi \alpha} \frac{3}{5}\left[\left(2 C_{1 u}-C_{1 d}\right)+\left(2 C_{2 u}-C_{2 d}\right) \frac{1-(1-y)^{2}}{1+(1-y)^{2}}\right]$,

where $Q^{2}=-q^{2}$ and $y=P q / P k$.

To include corrections from possible BSM and as well as other SM pieces, we can reparametrize the Cahn-Gilman formula [8]

$$
A_{\mathrm{RL}}=-\frac{G_{F} Q^{2}}{2 \sqrt{2} \pi \alpha} \frac{3}{5}\left[\tilde{a}_{1}+\tilde{a}_{2} \frac{1-(1-y)^{2}}{1+(1-y)^{2}}\right]
$$

with $\tilde{a}_{i}=-\left(2 C_{i u}-C_{i d}\right)\left(1+R_{i}\right)$. Here, $R_{i}$ describes any deviation of the $C_{i}$ from the expressions in Eqs. (3) to (6), including both SM and BSM corrections. In this paper we concentrate on $R_{1}^{\mathrm{HT}}$, namely the higher-twist correction to $\tilde{a}_{1}$.

Bjorken and Wolfenstein [22,23] showed that if one assumes isospin symmetry and neglects sea quark contributions, then there is only one matrix element that contributes to $R_{1}^{\mathrm{HT}}$ (for a detailed review of these arguments in a more modern context, see Ref. [8]). This observation significantly simplifies the theoretical interpretation of the asymmetry, 
allowing us to concentrate on one particular matrix element without needing to to disentangle the contributions from many different sources. In brief, the Bjorken and Wolfenstein argument works as follows: $A_{\mathrm{RL}}$ arises from the interference between the electromagnetic and weak neutral currents. First, one can decompose both currents into an isoscalar $S$ and an isovector $V$ term. The matrix elements of the $S \times V$ cross term vanishes because deuteron is an isosinglet. Furthermore, at leading twist, we have $\langle S S\rangle=\langle V V\rangle$. Therefore, the difference between $\langle S S\rangle$ and $\langle V V\rangle$ that enters hadronic tensor $W_{\mu \nu}$

$$
\begin{aligned}
& W_{u d}^{\mu v}(P, q) \\
& =\frac{1}{8 \pi M_{D}} \int d^{4} z e^{i q \cdot z}\langle D(P)| \bar{u}(z) \gamma^{\mu} u(z) \bar{d}(0) \gamma^{v} d(0) \\
& \quad+(u \leftrightarrow d)|D(P)\rangle
\end{aligned}
$$

with $M_{D}$ being the mass of deuteron, is the only matrix element giving a HT correction to $R_{1}$.

Below, we will compute the matrix element (9) using an expansion of string operators [24] in order to extract the twistfour piece; the latter is expressed in terms of the deuteron twistfour distribution function $\tilde{Q}_{D}\left(x_{B}\right)$, which will be computed in Sec. IV.

\section{LIGHT-CONE AMPLITUDES}

The main challenge in proceeding from (9) is our ignorance of the details of the nucleon wave functions. As QCD is nonperturbative at the hadronic scale, analytical expressions for the wave functions are unknown. At present, lattice QCD can provide only HT contributions to structure function moments and not the $x_{B}$ dependence of the $R_{1}^{\mathrm{HT}}$ that is of interest to the SoLID experiment. Consequently, one must turn to various models that seek to incorporate nonperturbative dynamics. Previous works on $R_{1}^{\mathrm{HT}}$ include the use of the MIT bag model [8] and isotropic light-cone wave functions that contain both quark and gluon Fock components [9]; their results yield similar shapes for the $x_{B}$ dependence but differ somewhat in magnitude, with a maximum $R_{1}^{\mathrm{HT}}$ of $0.003 \sim 0.005$ at $0.2<x_{B}<0.7$ for $Q^{2}=4 \mathrm{GeV}^{2}$, which is a little bit lower than the achievable precision level in the SoLID experiment.

In this work we study how the inclusion of additional parton angular momentum might modify the $R_{1}^{\mathrm{HT}}$ prediction. For this purpose, we adopt the formalism developed in Ref. [25], starting from a light-cone formulation of quark states, which is equivalent to the well-known "infinite momentum frame" point of view that gives the PDF its intuitive meaning as a parton momentum probability distribution [6]. We then perform a light-cone expansion of the nucleon state, retaining only the portion of Fock space containing three valence quarks with all possible quark OAM. To illustrate, we consider a spin-up proton. Its three valence quarks can form a total helicity of $\pm 1 / 2, \pm 3 / 2$; therefore in order to keep the total proton spin in the $z$ direction to be $1 / 2$ we need to assign different $z$ component quark OAM (i.e., $l_{z}$ ) for different combinations.

A spin-up proton state, then, can be parametrized as the follows:

$$
|P \uparrow\rangle=|P \uparrow\rangle^{l_{z}=0}+|P \uparrow\rangle^{l_{z}=1}+|P \uparrow\rangle^{l_{z}=-1}+|P \uparrow\rangle^{l_{z}=2}
$$

with

$$
\begin{aligned}
|P \uparrow\rangle^{l_{z}=0} & =\frac{\epsilon^{a b c}}{\sqrt{6}} \int\left[D X_{3}\right]\left(\psi^{(1)}(1,2,3)+i\left(k_{1}^{x} k_{2}^{y}-k_{1}^{y} k_{2}^{x}\right) \psi^{(2)}(1,2,3)\right) u_{a \uparrow}^{\dagger}(1)\left\{u_{b \downarrow}^{\dagger}(2) d_{c \uparrow}^{\dagger}(3)-d_{b \downarrow}^{\dagger}(2) u_{c \uparrow}^{\dagger}(3)\right\}|0\rangle \\
|P \uparrow\rangle^{l_{z}=1} & =\frac{\epsilon^{a b c}}{\sqrt{6}} \int\left[D X_{3}\right]\left(k_{1 \perp}^{+} \psi^{(3)}(1,2,3)+k_{2 \perp}^{+} \psi^{(4)}(1,2,3)\right)\left(u_{a \uparrow}^{\dagger}(1) u_{b \downarrow}^{\dagger}(2) d_{c \downarrow}^{\dagger}(3)-d_{a \uparrow}^{\dagger}(1) u_{b \downarrow}^{\dagger}(2) u_{c \downarrow}^{\dagger}(3)\right)|0\rangle \\
|P, \uparrow\rangle^{l_{z}=-1} & =\frac{\epsilon^{a b c}}{\sqrt{6}} \int\left[D X_{3}\right]\left(-k_{2 \perp}^{-} \psi^{(5)}(1,2,3)\right)\left(u_{a \uparrow}^{\dagger}(1) u_{b \uparrow}^{\dagger}(2) d_{c \uparrow}^{\dagger}(3)-u_{a \uparrow}^{\dagger}(1) d_{b \uparrow}^{\dagger}(2) u_{c \uparrow}^{\dagger}(3)\right)|0\rangle \\
|P \uparrow\rangle^{l_{z}=2} & =\frac{\epsilon^{a b c}}{\sqrt{6}} \int\left[D X_{3}\right] k_{1 \perp}^{+} k_{3 \perp}^{+} \psi^{(6)}(1,2,3)\left(u_{a \downarrow}^{\dagger}(1) d_{b \downarrow}^{\dagger}(2) u_{c \downarrow}^{\dagger}(3)-u_{a \downarrow}^{\dagger}(1) u_{b \downarrow}^{\dagger}(2) d_{c \downarrow}^{\dagger}(3)\right)|0\rangle,
\end{aligned}
$$

where $k_{i \perp}^{ \pm}=k_{i}^{x} \pm i k_{i}^{y}$, while $u_{a i}^{\dagger}(1)$ means the creation operator of an up-quark (same for down-quark) with color $a$, spin $i$ and momentum $k_{1}$, etc., satisfying the light-cone anticommutation relation

$$
\left\{u_{a i}(p), u_{b j}^{\dagger}\left(p^{\prime}\right)\right\}=2 p^{+}(2 \pi)^{3} \delta_{a b} \delta_{i j} \delta\left(p^{+}-p^{\prime+}\right) \delta^{(2)}\left(\vec{p}_{\perp}-\vec{p}_{\perp}^{\prime}\right) .
$$

The integration measure is ${ }^{1}$

$$
\begin{aligned}
\int\left[D X_{3}\right]= & \sqrt{2} \frac{d x_{1} d x_{2} d x_{3}}{\sqrt{2 x_{1} 2 x_{2} 2 x_{3}}} \frac{d^{2} \vec{k}_{1 \perp} d^{2} \vec{k}_{2 \perp} d^{2} \vec{k}_{3 \perp}}{(2 \pi)^{9}} \\
& \times 2 \pi \delta\left(1-x_{1}-x_{2}-x_{3}\right) \\
& \times(2 \pi)^{2} \delta^{(2)}\left(\vec{k}_{1 \perp}+\vec{k}_{2 \perp}+\vec{k}_{3 \perp}\right)
\end{aligned}
$$

The proton wave function amplitudes $\left\{\psi^{(1)} \ldots \psi^{(6)}\right\}$ are generally unknown functions. Although the expansion (11)-(14) is generic, the explicit form of $\psi^{(i)}$ is model dependent. In this work, we chose the form of $\psi^{(i)}$ derived in Ref. [18] by starting

\footnotetext{
${ }^{1}$ There might be difference in constant factors in the definition of integration measure by different authors, which only affects the overall normalization.
} 
from the static solution of a constituent quark model [26] (which works well in predicting many electroweak properties of the baryons) and applying a Melosh rotation to the solution to obtain nonzero $l_{z}$ components [27]. This choice of proton wave function is used to predict the first moment of Sivers function, and turns out to agree fairly well with the experimental measurements from HERMES and COMPASS $[28] .^{2}$

\section{MATRIX ELEMENTS BETWEEN NUCLEON STATES}

Following Ref. [24], in order to obtain the twist-four distribution function $\tilde{Q}_{D}(x)$ we need to evaluate the matrix elements between state $|D(P)\rangle$ of the following operators:

$$
\begin{aligned}
& Q_{A}(b, z) \equiv: \bar{u}\left(b_{1} z\right) t^{a} \nless \gamma_{5} u\left(b_{2} z\right) \bar{d}\left(b_{3} z\right) t^{a} \nless \gamma_{5} d\left(b_{4} z\right): \\
& Q_{V}(b, z) \equiv: \bar{u}\left(b_{1} z\right) t^{a} \nless u\left(b_{2} z\right) \bar{d}\left(b_{3} z\right) t^{a} \nless d\left(b_{4} z\right):,
\end{aligned}
$$

where $z$ is a coordinate on light cone, and the parameters $b \equiv$ $\left\{b_{1}, b_{2}, b_{3}, b_{4}\right\}$ characterize the light-cone separation between quark field operators.

When computing the matrix elements of $Q_{V, A}$ in Eq. (17) we assume an incoherent impulse approximation in which the incoming photon strikes only one of the two nucleons (see, e.g., Ref. [29] for further discussions regarding the impulse approximation); hence, matrix elements of the operators (17) can be related to the same matrix elements taken between proton states (or equivalently between neutron states, given isospin symmetry). Also, since the quantities we compute do not depend on the proton spin, we can take it to be $+1 / 2$ along the $z$ direction without loss of generality.

Now, starting from the operators (17), we define two distribution functions $Q_{ \pm}\left(x_{\xi}\right)$ via

$$
\begin{aligned}
& \left\langle P(p) \uparrow\left|\left\{Q_{V}(b, z) \pm Q_{A}(b, z)\right\}\right| P(p) \uparrow\right\rangle \\
& \equiv(p \cdot z)^{2} \int \prod_{k=1}^{4} d x_{\xi_{k}} \delta\left(\sum_{i} x_{\xi_{i}}\right) e^{-i(p \cdot z) \sum_{k} b_{k} x_{\xi_{k}}} Q_{ \pm}\left(x_{\xi}\right)
\end{aligned}
$$

with $x_{\xi}$ collectively representing $\left\{x_{\xi_{1}}, x_{\xi_{2}}, x_{\xi_{3}}, x_{\xi_{4}}\right\}$, the lightcone momentum fractions: $\xi_{i}^{+}=x_{\xi_{i}} p^{+}$. Meanwhile $|P(p) \uparrow\rangle$ is the spin-up proton state with momentum $p$. Substituting (11)-(14) into (18) we are able to express $Q_{ \pm}\left(x_{\xi}\right)$ in terms of the proton wave function amplitudes. It is easy to observe that only diagonal terms (i.e., terms with the same $l_{z}$ in initial and final states) could give nonvanishing contributions. After a rather lengthy derivation with the aid of Eq. (A2), we obtain

$$
\begin{aligned}
Q_{ \pm}\left(x_{\xi}\right)= & -\frac{32 \pi^{3}}{3} \int \frac{d^{2} \vec{\xi}_{1 \perp}}{(2 \pi)^{3}} \ldots \frac{d^{2} \vec{\xi}_{4 \perp}}{(2 \pi)^{3}} \\
& \times \theta\left(-x_{\xi_{1}}\right) \theta\left(x_{\xi_{2}}\right) \theta\left(-x_{\xi_{3}}\right) \theta\left(x_{\xi_{4}}\right) \theta\left(1-x_{\xi_{2}}-x_{\xi_{4}}\right) \\
& \times \delta^{2}\left(\vec{\xi}_{1 \perp}+\vec{\xi}_{2 \perp}+\vec{\xi}_{3 \perp}+\vec{\xi}_{4 \perp}\right) \\
& \times \sum_{l_{z}} \psi_{l_{z}}^{ \pm}\left(-\xi_{1},-\xi_{3}, \xi_{2}, \xi_{4}\right)
\end{aligned}
$$

where the explicit formulas of $\psi_{l_{2}}^{ \pm}$are given in Appendix B.

\footnotetext{
${ }^{2}$ References [18] and [28] defined their first moment of Sivers function with a sign difference.
}

The proton twist-four distribution function can now be expressed in terms of the $Q_{ \pm}$(refer to Eq. 42 of Ref. [9] after some rearrangement)

$$
\begin{aligned}
\tilde{Q}_{p}\left(x_{B}\right) \equiv & 2 \operatorname{Re} \int_{-1}^{1} \frac{\prod_{k=1}^{4} d x_{\xi_{k}}}{x_{\xi_{2}} x_{\xi_{3}}\left(x_{\xi_{2}}+x_{\xi_{3}}\right)} \delta\left(\sum_{k} x_{\xi_{k}}\right) \\
& \times\left\{\left(x_{\xi_{2}}+x_{\xi_{3}}\right) \delta\left(x_{B}+x_{\xi_{1}}+x_{\xi_{2}}\right)-x_{\xi_{3}} \delta\left(x_{B}+x_{\xi_{1}}\right)\right. \\
& \left.-x_{\xi_{2}} \delta\left(x_{\xi_{4}}-x_{B}\right)\right\}\left[\left(1+P_{14} P_{23}\right) Q_{+}\left(x_{\xi}\right)\right. \\
& \left.-\left(P_{12}+P_{34}\right) Q_{-}\left(x_{\xi}\right)\right] .
\end{aligned}
$$

Here $P_{i j}$ is the permutation operator, e.g., $P_{12} Q_{+}\left(x_{\xi_{1}}, x_{\xi_{2}}, x_{\xi_{3}}, x_{\xi_{4}}\right)=Q_{+}\left(x_{\xi_{2}}, x_{\xi_{1}}, x_{\xi_{3}}, x_{\xi_{4}}\right)$. The deuteron twist-four distribution function $\tilde{Q}_{D}\left(x_{B}\right)$ can be expressed in terms of $\tilde{Q}_{p}\left(x_{B}\right)$ through an incoherent impulse approximation [30], which says that a general deuteron hadronic tensor can be related to the corresponding hadronic tensors of proton and neutron by

$$
M_{D} W_{D}^{\mu \nu}(p, q) \approx M_{N} W_{p}^{\mu \nu}\left(\frac{p}{2}, q\right)+M_{N} W_{n}^{\mu \nu}\left(\frac{p}{2}, q\right),
$$

where $M_{N}$ is the mass of nucleon. In the equation above each hadronic tensor is multiplied by the particle's mass, because following Eq. (9) the hadronic tensor we defined has dimension -1 . Now we can express both sides of Eq. (21) in terms of dimensionless structure functions $\left\{F_{i}\left(x_{B}\right)\right\}$. Using isospin symmetry and the fact that $\tilde{Q}\left(x_{B}\right)$ is proportional to $x_{B}^{-1} F_{1}^{u d}\left(x_{B}\right)$ (see Eq. 34 of Ref. [9]), we obtain ${ }^{3}$

$$
\frac{1}{2} \tilde{Q}_{D}\left(x_{B} / 2\right) \approx \tilde{Q}_{p}\left(x_{B}\right)+\tilde{Q}_{n}\left(x_{B}\right) \approx 2 \tilde{Q}_{p}\left(x_{B}\right) .
$$

Finally, following the logic of Ref. [9], one can the derive the twist-four contribution to $R_{1}$

$$
R_{1}^{\mathrm{HT}}\left(x_{B}, Q^{2}\right)=\frac{1}{Q^{2}} \frac{\alpha_{s} \pi}{5\left(1-\frac{20}{9} \sin ^{2} \theta_{W}\right)} \frac{x_{B} \tilde{Q}_{D}\left(x_{B}\right)}{u_{D}\left(x_{B}\right)+d_{D}\left(x_{B}\right)}
$$

with $q_{D}\left(x_{B}\right)$ being the parton distribution function for quark of flavor $q$ in the deuteron

$$
\langle D(P)|\bar{q}(z) \nless q(-z)| D(P)\rangle=2(P \cdot z) \int_{-1}^{1} d x e^{2 i(P \cdot z) x} q_{D}(x) .
$$

Note that we neglect the logarithmic $Q^{2}$ dependence of the structure functions in this analysis. We can express $q_{D}$ in terms of PDF of the proton and neutron again by the impulse approximation (21), but now comparing the structure function $F_{2}\left(x_{B}\right)$ on both sides, which is proportional to $x_{B}^{-1} q\left(x_{B}\right)$. The

\footnotetext{
${ }^{3}$ In Ref. [9], the authors did not multiply their hadronic tensors by particle mass in the impulse approximation formula, therefore the corresponding relation they obtained is off by a factor $1 / 2$; same for the relation of quark distribution functions.
} 
result is

$$
q_{D}\left(x_{B} / 2\right) \approx q_{p}\left(x_{B}\right)+q_{n}\left(x_{B}\right)
$$

where $q_{p}(x)$ and $q_{n}(x)$ are defined as in Eq. (24) but for proton/neutron states. Furthermore, neglecting CSV effects we have

$$
u_{n}\left(x_{B}\right)=d_{p}\left(x_{B}\right), \quad d_{n}\left(x_{B}\right)=u_{p}\left(x_{B}\right) .
$$

Therefore, it is sufficient to just calculate $u_{p}\left(x_{B}\right)$ and $d_{p}\left(x_{B}\right)$ using the proton light-cone wave function (11)-(14). Using (A3) and (A4), we can compute the quark PDFs of the (spin-up) nucleons by calculating the matrix element on LHS of Eq. (24) with nucleon states, and compare it with the form on RHS to extract the PDFs. Same with the twist-four distribution functions, only terms diagonal to $l_{z}$ survive, so we can separate the result into components of different $l_{z}$ as the following:

$$
\begin{aligned}
u_{p}\left(x_{B}\right)+d_{p}\left(x_{B}\right)= & d_{n}\left(x_{B}\right)+u_{n}\left(x_{B}\right) \\
= & \frac{1}{(2 \pi)^{6}} \int_{0}^{1} d x_{1} \int d^{2} \vec{k}_{1 \perp} d^{2} \vec{q}_{\perp} \\
& \times \Theta\left(1-x_{B}-x_{1}\right) \sum_{l_{z}} A^{l_{z}}(q, 1,2),
\end{aligned}
$$

where the functions $A^{l_{z}}(q, 1,2)$ are given in Appendix B.

We now proceed to show that a partial cancelation occurs between contributions of $l_{z}=+1$ and $l_{z}=-1$. For this purpose, we combine (19) and (20), together with the fact that $\psi_{l_{z}}^{ \pm}\left(q, l, q^{\prime}, l^{\prime}\right)^{*}=\psi_{l_{z}}^{ \pm}\left(q^{\prime}, l^{\prime}, q, l\right)$, to simplify the expression of $\tilde{Q}_{p}\left(x_{B}\right)$ as

$$
\tilde{Q}_{p}\left(x_{B}\right)=\tilde{Q}_{p}^{+}\left(x_{B}\right)+\tilde{Q}_{p}^{-}\left(x_{B}\right),
$$

where

$$
\begin{aligned}
\tilde{Q}_{p}^{+}\left(x_{B}\right)= & \frac{64 \pi^{3}}{3} \int_{0}^{1} \prod_{i=1}^{4} d x_{\xi_{i}} \delta\left(x_{\xi_{1}}-x_{\xi_{2}}+x_{\xi_{3}}-x_{\xi_{4}}\right) \theta\left(1-x_{\xi_{2}}-x_{\xi_{4}}\right)\left\{\frac{\delta\left(x_{B}-x_{\xi_{1}}+x_{\xi_{2}}\right)}{x_{\xi_{2}} x_{\xi_{3}}}+\frac{\delta\left(x_{B}-x_{\xi_{1}}\right)}{x_{\xi_{2}}\left(x_{\xi_{2}}-x_{\xi_{3}}\right)}-\frac{\delta\left(x_{B}-x_{\xi_{4}}\right)}{x_{\xi_{3}}\left(x_{\xi_{2}}-x_{\xi_{3}}\right)}\right. \\
& \left.+\frac{\delta\left(x_{B}+x_{\xi_{3}}-x_{\xi_{4}}\right)}{x_{\xi_{4}} x_{\xi_{2}}}\right\} \int \prod_{i=1}^{4} \frac{d^{2} \vec{\xi}_{i \perp}}{(2 \pi)^{3}} \delta^{2}\left(\vec{\xi}_{\perp 1}-\vec{\xi}_{\perp 2}+\vec{\xi}_{\perp 3}-\vec{\xi}_{\perp 4}\right) \sum_{l_{z}} \operatorname{Re} \psi_{l_{z}}^{+}\left(\xi_{1}, \xi_{3}, \xi_{2}, \xi_{4}\right) \\
\tilde{Q}_{p}^{-}\left(x_{B}\right)= & \frac{64 \pi^{3}}{3} \int_{0}^{1} \prod_{i=1}^{4} d x_{\xi_{i}} \delta\left(x_{\xi_{1}}-x_{\xi_{2}}-x_{\xi_{3}}+x_{\xi_{4}}\right) \theta\left(1-x_{\xi_{2}}-x_{\xi_{3}}\right)\left\{\frac{\delta\left(x_{B}+x_{\xi_{1}}-x_{\xi_{2}}\right)}{x_{\xi_{2}} x_{\xi_{3}}}-\frac{\delta\left(x_{B}-x_{\xi_{4}}\right)}{x_{\xi_{3}}\left(x_{\xi_{2}}+x_{\xi_{3}}\right)}-\frac{\delta\left(x_{B}-x_{\xi_{1}}\right)}{x_{\xi_{2}}\left(x_{\xi_{2}}+x_{\xi_{3}}\right)}\right. \\
& \left.+\frac{\delta\left(x_{B}-x_{\xi_{1}}+x_{\xi_{2}}\right)}{x_{\xi_{2}} x_{\xi_{3}}}\right\} \int \prod_{i=1}^{4} \frac{d^{2} \vec{\xi}_{i \perp}}{(2 \pi)^{3}} \delta^{2}\left(\vec{\xi}_{\perp 1}-\vec{\xi}_{\perp 2}-\vec{\xi}_{\perp 3}+\vec{\xi}_{\perp 4}\right) \sum \sum_{l_{z}} \operatorname{Re} \psi_{l_{z}}^{-}\left(\xi_{2}, \xi_{3}, \xi_{1}, \xi_{4}\right)
\end{aligned}
$$

First we qualitatively analyze the contribution from each $l_{z}$ component to $\tilde{Q}_{p}^{ \pm}\left(x_{B}\right)$. This can be done by simply referring to Eqs. (B1)-(B8) of the Appendix B. The result is summarized in Table I. We observe that the $l_{z}=+1(-1)$ piece contributes mainly to $\tilde{Q}_{p}^{-}\left(\tilde{Q}_{p}^{+}\right)$. Also notice that we do not include the $l_{z}=2$ component as its effect is tiny.

Next we study the behavior of different contributions to $\tilde{Q}_{p}^{ \pm}\left(x_{B}\right)$ with respect to $x_{B}$, showing that those associated with the $l_{z} \pm 1$ components largely cancel. The individual contributions from the latter are shown in the top two panels of Fig. 2. We observe that the $l_{z}=-1$ contribution, which contributes only to $\tilde{Q}_{p}^{+}\left(x_{B}\right)$ changes sign at $x_{B} \approx 0.4$, whereas

TABLE I. The contributions from different $l_{z}$ components to $\tilde{Q}_{p}^{ \pm}\left(x_{B}\right)$. The $l_{z}=0,+1$ components contribute mostly to $\tilde{Q}_{p}^{-}$ ("dominant") and less so to $\tilde{Q}_{p}^{+}$("subdominant"), while the $l_{z}=-1$ component contributes only to $\tilde{Q}_{p}^{+}$.

\begin{tabular}{lcl}
\hline \hline$l_{z}$ & Contribution to $\tilde{Q}_{p}^{+}\left(x_{B}\right)$ & Contribution to $\tilde{Q}_{p}^{-}\left(x_{B}\right)$ \\
\hline 0 & subdominant & dominant \\
+1 & subdominant & dominant \\
-1 & all & zero \\
\hline \hline
\end{tabular}

the $l_{z}=+1$ contribution does not. Consequently, the two contributions will cancel against each other for $x_{B} \gtrsim 0.4$. While the cancellation is not exact, it becomes more effective at larger values of $x_{B}$, a region that is weighted most strongly in $R_{1}^{\mathrm{HT}}$ by the factor of $x_{B}$ in the numerator of Eq. (23) and the corresponding presence of $u_{D}\left(x_{B}\right)+d_{D}\left(x_{B}\right)$ in the denominator.

We also note that this sign change and cancellation appears to be rather generic. To see why, let us naively take

$$
\int \prod_{i=1}^{4} \frac{d^{2} \vec{\xi}_{i \perp}}{(2 \pi)^{3}} \delta^{2}(\ldots) \operatorname{Re} \psi_{l_{z}}^{ \pm} \approx \text { constant } \equiv C,
$$

assuming the function above is well-behaved with respect to $\left\{x_{\xi_{i}}\right\}$. This approximation simply means that we do not care about the details of the proton wave function amplitudes. Under this approximation, the numerical integration (29) and (30) can be performed quite trivially, and the result is shown in the lower two panels of Fig. 2. In this case, we show $\tilde{Q}_{p}^{ \pm}\left(x_{B}\right)$ as the $l_{z}= \pm 1$ components contribute primarily to one or the other of these two quantities (see Table I). Although the the assumption in Eq. (31) breaks down at large and small $x_{B}$, one can see that a sign change of $\tilde{Q}_{p}^{+}\left(x_{B}\right)$ from negative to positive 

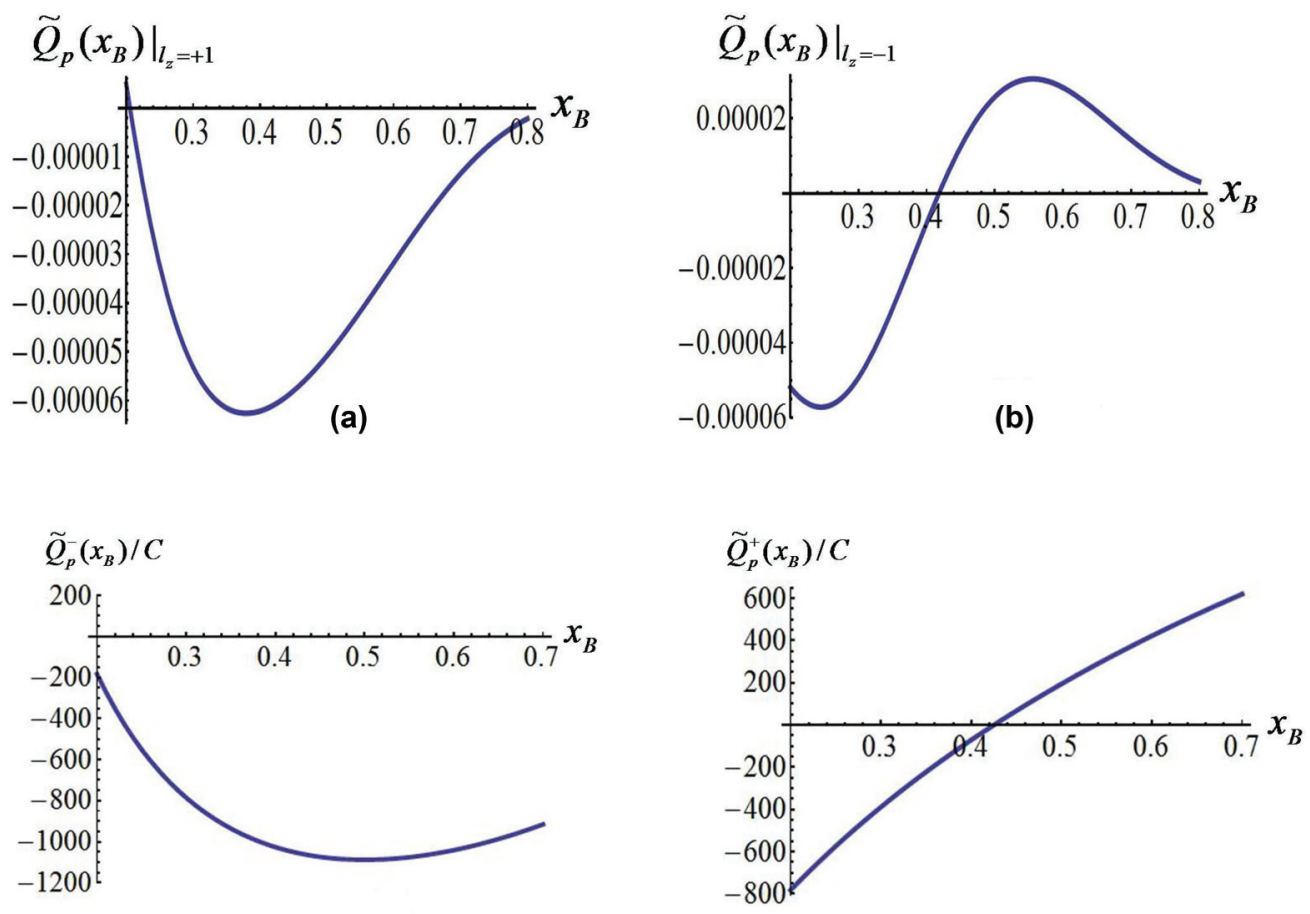

(c)

(d)

FIG. 2. (Color online) Top panels: full results for $l_{z} \pm 1$ contributions to $\tilde{Q}_{p}\left(x_{B}\right)$. Bottom panels: behavior of $\tilde{Q}_{p}^{\mp}\left(x_{B}\right)$ ignoring the details of nucleon wave function amplitudes. The constant $\mathrm{C}$ is defined in Eq. (31).

occurs near $x_{B}=0.4$, implying that $\tilde{Q}_{p}^{+}\left(x_{B}\right)$ and $\tilde{Q}_{p}^{-}\left(x_{B}\right)$ will have different signs for $x_{B} \gtrsim 0.4$. Therefore, according to Table I, the contribution to $\tilde{Q}_{p}\left(x_{B}\right)$ from $l_{z}=1$ and $l_{z}=-1$ should partially cancel other for $x_{B} \gtrsim 0.4$. Furthermore, since the argument above does not depend on the details of the nucleon wave function (as long as it is well behaved), this feature of partial cancellation should be generic.

\section{NUMERICAL RESULTS AND DISCUSSION}

Equations (29) and (30) are our starting point for the numerical evaluation of $\tilde{Q}_{p}\left(x_{B}\right)$, which involves an eightfold integration. To perform this integration, we adopt the Monte Carlo numerical integration called Divonne contained in the CUBA Library, which is an algorithm package designed for multidimensional numerical integration [31]. For each $l_{z}$ component, we evaluate the value of $\tilde{Q}_{p}\left(x_{B}\right)$ at a series of discrete $\left\{x_{B, i}\right\}$, and then link them together using a best-fit line. Also, we take $\alpha_{s}=0.5$ at $1 \mathrm{GeV}$ following the renormalization group (RG) prediction of the running coupling constant at four-loop order together with a three-loop threshold matching, with the quark thresholds taken to be $M_{c}=1.5 \mathrm{GeV}$ and $M_{b}=4.7 \mathrm{GeV}$ respectively [32].

Our main result is shown in Fig. 3, which gives $R_{1}^{\mathrm{HT}}$ versus $x_{B}^{\prime} \equiv 2 x_{B}$ at $Q^{2}=4 \mathrm{GeV}^{2}$. First, let us compare this outcome with that of Refs. [8,9]. It turns out that all three calculations predict similar curve shape for $R_{1}^{\mathrm{HT}}$, only with slightly different positions of peak and zero-point. Concerning the magnitude, our work predicts a maximum absolute value $\left|R_{1}^{\mathrm{HT}}\right| \approx 2.6 \times$ $10^{-3}$ between $0.2<x_{B}^{\prime}<0.7$, which is smallest in magnitude among all the three predictions, and is about a half of the size to that of Ref. [9]. This is understandable because the authors include a three-quark +1 -gluon Fock-space component whose contribution is comparable in magnitude to that of the pure three-quark state. Nonetheless, all three calculations suggest that $\left|R_{1}^{\mathrm{HT}}\right|$ lies below that of the expected SoLID precision.

Next we study the OAM dependence in detail. To that end, we first introduce some nomenclature: in the following, we will use the notation $\left(\left|l_{z}\right| \otimes\left|l_{z^{\prime}}\right|\right)$, which denotes a generic matrix element taking between two hadronic states, of which one of them has absolute value of quark OAM in the $z$ direction being $\left|l_{z}\right|$ and the other being $\left|l_{z^{\prime}}\right|$.

From our arguments at the end of Sec. IV, we expect that although $l_{z}= \pm 1$ individually contribute a significant amount to $\tilde{Q}_{p}\left(x_{B}\right)$, they should largely cancel against each other for

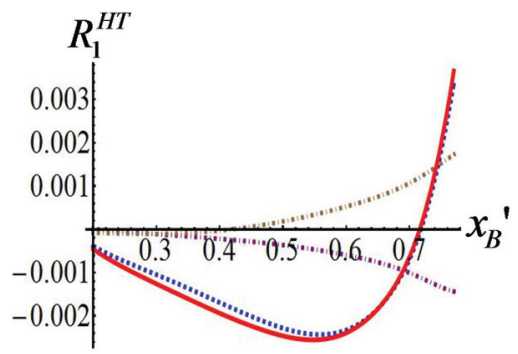

FIG. 3. (Color online) The twist-four correction to $R_{1}$ at $Q^{2}=$ $4 \mathrm{GeV}^{2}$. The blue dashed curve shows the $l_{z}=0$ contribution; purple dot-dashed curve shows the $l_{z}=1$ contribution; brown dot-dashed curve shows the $l_{z}=-1$ contribution; the red solid curve is the sum of all. $l_{z}=2$ contribution is negligible and therefore not included. 


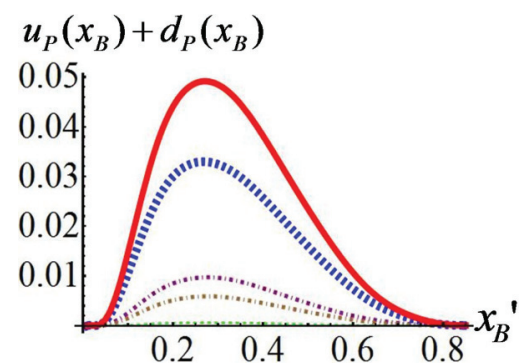

FIG. 4. (Color online) The unnormalized QDF of spin-up proton, split into contributions from different $l_{z}$ components. Blue thickdashed curve shows contribution from $l_{z}=0$ component; purple dot-dashed curve shows contribution from $l_{z}=1$ component; brown dot-dashed curve shows contribution from $l_{z}=-1$ component; green thin-dashed curve shows contribution from $l_{z}=2$ component; red solid curve is the sum of all contributions.

$x_{B}>0.4$, making the total $(1 \otimes 1)$ contribution rather small, and therefore leaving the $(0 \otimes 0)$ contribution as the dominant piece. This expectation is born out by the curves in Fig. 3. The purple dot-dashed curve and brown dot-dashed curves give the individual $\left(l_{z}=1\right) \otimes\left(l_{z}=1\right)$ and $\left(l_{z}=-1\right) \otimes\left(l_{z}=-1\right)$ contributions, respectively, which exhibit the expected cancellation for $x_{B}^{\prime}>0.4$. The blue dashed curve and red solid curve give the $(0 \otimes 0)$ and total contributions, respectively. It is clear that the former dominates the total. This $(0 \otimes 0)$ dominance is a rather unique feature of the particular twist-four contribution of interest here, and one that is not shared by other diagonal matrix elements. For example, if one calculate proton quark PDFs (leading twist) using the same set of wave functions, the $(0 \otimes 0)$ and $(1 \otimes 1)$ contributions are comparable; moreover, since they have the same sign, the two $\left|l_{z}\right|=1$ pieces do not cancel each other (see Fig. 4).

On the other hand, we also note that there are hadronic matrix elements that depend crucially on the existence of nonzero quark OAM in light-cone quantization. In particular, in Ref. [18], the authors studied the Sivers function [33] and Boer-Mulders function [34], which are examples of transverse momentum dependent parton distribution functions (TMDs), appearing in semi-inclusive deep-inelastic scattering. Importantly, both distribution functions depend on off-diagonal matrix elements of $l_{z}$ : the Sivers function is sensitive to $(0 \otimes 1)$ while Boer-Mulders function is sensitive to both $(0 \otimes 1)$ and $(1 \otimes 2)$. Simply speaking, the existence of nonzero quark OAM is responsible for the nonvanishing values of the Sivers and Boer-Mulders functions. Combining this observation with our analysis of the HT matrix element, we conclude that the

TABLE II. The dependence on different quark light-cone OAM components of various distribution functions.

\begin{tabular}{lcc}
\hline \hline Distribution functions & $\begin{array}{c}\text { Dominant } \\
\text { contribution(s) }\end{array}$ & $\begin{array}{c}\text { Subdominant } \\
\text { contribution(s) }\end{array}$ \\
\hline Quark distribution functions & $(0 \times 0),(1 \otimes 1)$ & $(2 \otimes 2)$ \\
PVDIS twist-four correction & $(0 \otimes 0)$ & $(1 \otimes 1),(2 \otimes 2)$ \\
Sivers function & $(0 \otimes 1)$ & $(1 \otimes 2)$ \\
Boer-Mulders function & $(0 \otimes 1),(1 \otimes 2)$ & - \\
\hline \hline
\end{tabular}

twist-four correction to $e \mathrm{D}$ PVDIS is essentially transparent to the parton angular momentum dynamics that generate the Sivers and Boer-Mulders functions.

It is also interesting to study the impact of sea-parton dynamics on the behavior of the HT matrix element. To that end, we performed a qualitative analysis of the contribution made by the Fock space component containing three quarks + one gluon, using the general form suggested in Ref. [35] that includes nonzero gluon OAM. The authors of Ref. [9] computed the contribution of the $3 q+1 g$ state with $l_{z}=0$, which turns out to have a similar shape to that of the $l_{z}=03 q$-state contribution. To our knowledge, however, there exist no explicit functional forms for the $3 q+1 g$ nucleon wave function with nonzero parton OAM. Consequently, our analysis is purely analytic at this point. We observe that, in contrast to the $3 q$ state contribution, the matrix element of $3 q+1 g$ state for a fixed $l_{z}$ can contribute significantly to both $\tilde{Q}_{p}^{ \pm}\left(x_{B}\right)$ simultaneously; therefore there is no obvious correlation between $l_{z}$ and $\tilde{Q}_{p}^{ \pm}\left(x_{B}\right)$ and hence no obvious pattern of partial cancellation. In Table II we summarize the importance of different $\left(\left|l_{z}\right| \otimes\left|l_{z}^{\prime}\right|\right)$ contributions to various distribution functions, considering only the contributions of $3 q$ states.

Combining observations, we may draw the following conclusion: if a future $e \mathrm{D}$ PVDIS measurement yields a sufficiently precise determination of $R_{1}^{\mathrm{HT}}$ as a function of $x_{B}^{\prime}$, one can compare the experimental curve with our current theoretical prediction. A significant deviation from the predicted curve (e.g., the peak and zero-point are shifted by a considerable amount), could signal the importance of parton angular momentum dynamics beyond those responsible for the Sivers, Boer-Mulders, and spin-independent parton distribution functions.

\section{SUMMARY}

The next generation of parity-violating electron-scattering experiments are poised to probe both possible BSM physics as well as novel features of hadron and nuclear structure. In this work, we have studied one particular hadronic effect, namely, the twist-four contribution to $\tilde{a}_{1}$, the $y$-independent term in the $\mathrm{PV}$ asymmetry. Using a set of proton light-cone wave functions with nonzero quark orbital angular momentum, we evaluated the twist-four contribution as a function of $x_{B}$, identifying the contributions from different OAM-components. Our total for the correction $R_{1}^{\mathrm{HT}}$ is similar in both shape and magnitude to those obtained in previous works, indicating that higher precision than expected with the SoLID experiment would be needed to discern this HT effect. An effort to achieve such precision may be worthwhile, because $R_{1}^{\mathrm{HT}}$ appears to be rather unique, in the sense that it is not significantly affected by the parton angular momentum physics responsible for the existence of some other DIS observables such as the Sivers and Boer-Mulders functions. Thus, by combining the results of a more precise measurement of the asymmetry with measurements of other distribution functions, it is possible to probe complementary aspects of parton angular momentum and, perhaps, shed new light on the role of angular momentum in the structure of the nucleon. 


\section{ACKNOWLEDGMENTS}

We would like to thank A. Belitksy for useful discussions and F. Yuan and B. Pasquini for pointing us to explicit examples of the nucleon wave function amplitudes. We are also grateful to F. Yuan for a careful reading of the manuscript. This work is supported in part by DOE Contract No. DE-FG02-08ER41531 and by the Wisconsin Alumni Research Foundation.

\section{APPENDIX A: MATRIX ELEMENTS OF TWO- AND FOUR-FERMION OPERATORS}

In this section we present matrix elements of two-fermion operators $\left(u^{\dagger} u\right.$ and $\left.d^{\dagger} d\right)$ and four-fermion operators $\left(u^{\dagger} u d^{\dagger} d\right)$ between nucleon states. For this purpose let us consider two arbitrary components of proton light-cone wave function defined as the following:

$$
\begin{aligned}
\left|\psi_{\alpha}\right\rangle & \equiv \frac{\epsilon^{a b c}}{\sqrt{6}} \int\left[D X_{3}\right] \psi_{\alpha}(1,2,3) u_{a \lambda_{1}}^{\dagger}(1) u_{b \lambda_{2}}^{\dagger}(2) d_{c \lambda_{3}}^{\dagger}(3)|0\rangle \\
\left|\psi_{\beta}\right\rangle & \equiv \frac{\epsilon^{a b c}}{\sqrt{6}} \int\left[D X_{3}\right] \psi_{\beta}(1,2,3) u_{a \lambda_{1}^{\prime}}^{\dagger}(1) u_{b \lambda_{2}^{\prime}}^{\dagger}(2) d_{c \lambda_{3}^{\prime}}^{\dagger}(3)|0\rangle
\end{aligned}
$$

It is straightforward to work out the matrix elements of the four-fermion operator between these two states (the symbol " 1 " denotes the four-momentum $k_{1}=\left(x_{1} p^{+}, \vec{k}_{1 \perp}\right)$, which is given by $x_{1}=1-x_{q}-x_{l}=1-x_{q}^{\prime}-x_{l}^{\prime}$ and $\left.\vec{k}_{1 \perp}=-\vec{q}_{\perp}-\vec{l}_{\perp}=-\vec{q}_{\perp}^{\prime}-\vec{l}_{\perp}^{\prime}\right)$

$$
\begin{aligned}
& \left\langle\psi_{\alpha}\left|u_{i \rho}^{\dagger}(q) u_{i^{\prime} \rho^{\prime}}\left(q^{\prime}\right) d_{j \lambda}^{\dagger}(l) d_{j^{\prime} \lambda^{\prime}}\left(l^{\prime}\right)\right| \psi_{\beta}\right\rangle=\frac{32 \pi^{3}}{3}\left(\delta_{i i^{\prime}} \delta_{j j^{\prime}}-\delta_{i j^{\prime}} \delta_{i^{\prime} j}\right) \delta_{\lambda_{3} \lambda} \delta_{\lambda_{3}^{\prime} \lambda^{\prime}} \sqrt{x_{q} x_{l} x_{q}^{\prime} x_{l}^{\prime}} \delta\left(x_{q}+x_{l}-x_{q}^{\prime}-x_{l}^{\prime}\right) \delta^{2}\left(\vec{q}_{\perp}+\vec{l}_{\perp}-\vec{q}_{\perp}^{\prime}-\vec{l}_{\perp}\right) \\
& \times \int d x_{1} d^{2} \vec{k}_{1 \perp} \delta\left(1-x_{1}-x_{q}-x_{l}\right) \delta^{2}\left(\vec{k}_{1 \perp}+\vec{q}_{\perp}+\vec{l}_{\perp}\right)\left(\delta_{\lambda_{1} \rho} \delta_{\lambda_{2} \lambda_{2}^{\prime}} \delta_{\rho^{\prime} \lambda_{1}^{\prime}} \psi_{\alpha}^{*}(q, 1, l) \psi_{\beta}\left(q^{\prime}, 1, l^{\prime}\right)\right. \\
& +\delta_{\lambda_{1} \lambda_{2}^{\prime}} \delta_{\lambda_{2} \rho} \delta_{\rho^{\prime} \lambda_{1}^{\prime}} \psi_{\alpha}^{*}(1, q, l) \psi_{\beta}\left(q^{\prime}, 1, l^{\prime}\right)+\delta_{\lambda_{1} \rho} \delta_{\lambda_{2} \lambda_{1}^{\prime}} \delta_{\rho^{\prime} \lambda_{2}^{\prime}} \psi_{\alpha}^{*}(q, 1, l) \psi_{\beta}\left(1, q^{\prime}, l^{\prime}\right) \\
& \left.+\delta_{\lambda \lambda_{1}^{\prime}} \delta_{\lambda_{2} \rho} \delta_{\rho^{\prime} \lambda_{2}^{\prime}} \psi_{\alpha}^{*}(1, q, l) \psi_{\beta}\left(1, q^{\prime}, l^{\prime}\right)\right)
\end{aligned}
$$

and those for two-fermion operators,

$$
\begin{aligned}
\left\langle\psi_{\alpha}\left|d_{j \lambda}^{\dagger}(l) d_{j^{\prime} \lambda^{\prime}}\left(l^{\prime}\right)\right| \psi_{\beta}\right\rangle= & \frac{4}{3} x_{l} \delta\left(x_{l}-x_{l}^{\prime}\right) \delta^{2}\left(\vec{l}_{\perp}-\vec{l}_{\perp}^{\prime}\right) \delta_{\lambda_{3} \lambda} \delta_{\lambda_{3}^{\prime} \lambda^{\prime}} \delta_{j j^{\prime}} \int d x_{1} d x_{2} d^{2} \vec{k}_{1 \perp} d^{2} \vec{k}_{2 \perp} \delta\left(1-x_{1}-x_{2}-x_{l}\right) \\
& \times \delta^{2}\left(\vec{k}_{1 \perp}+\vec{k}_{2 \perp}+\vec{l}_{\perp}\right)\left(\delta_{\lambda_{1} \lambda_{1}^{\prime}} \delta_{\lambda_{2} \lambda_{2}^{\prime}} \psi_{\beta}(1,2, l)+\delta_{\lambda_{1} \lambda_{2}^{\prime}} \delta_{\lambda_{2} \lambda_{1}^{\prime}} \psi_{\beta}(2,1, l)\right) \psi_{\alpha}^{*}(1,2, l) \\
\left\langle\psi_{\alpha}\left|u_{j \lambda}^{\dagger}(l) u_{j^{\prime} \lambda^{\prime}}\left(l^{\prime}\right)\right| \psi_{\beta}\right\rangle= & \frac{4}{3} x_{l} \delta\left(x_{l}-x_{l}^{\prime}\right) \delta^{2}\left(\vec{l}_{\perp}-\vec{l}_{\perp}^{\prime}\right) \delta_{\lambda_{3} \lambda_{3}^{\prime}} \delta_{j j^{\prime}} \int d x_{1} d x_{2} d^{2} \vec{k}_{1 \perp} d^{2} \vec{k}_{2 \perp} \delta\left(1-x_{1}-x_{2}-x_{l}\right) \\
& \times \delta^{2}\left(\vec{k}_{1 \perp}+\vec{k}_{2 \perp}+\vec{l}_{\perp}\right)\left(\delta_{\lambda_{1} \lambda} \delta_{\lambda_{2} \lambda_{2}^{\prime}} \delta_{\lambda^{\prime} \lambda_{1}^{\prime}} \psi_{\alpha}^{*}(l, 1,2) \psi_{\beta}(l, 1,2)+\delta_{\lambda_{1} \lambda_{2}^{\prime}} \delta_{\lambda_{2} \lambda} \delta_{\lambda^{\prime} \lambda_{1}^{\prime}} \psi_{\alpha}^{*}(1, l, 2) \psi_{\beta}(l, 1,2)\right. \\
& \left.+\delta_{\lambda_{1} \lambda} \delta_{\lambda_{2} \lambda_{1}^{\prime}} \delta_{\lambda^{\prime} \lambda_{2}^{\prime}} \psi_{\alpha}^{*}(l, 1,2) \psi_{\beta}(1, l, 2)+\delta_{\lambda_{1} \lambda_{1}^{\prime}} \delta_{\lambda_{2} \lambda} \delta_{\lambda^{\prime} \lambda_{2}^{\prime}} \psi_{\alpha}^{*}(1, l, 2) \psi_{\beta}(1, l, 2)\right) .
\end{aligned}
$$

\section{APPENDIX B: COMPLETE FORMULAS FOR QUARK PDFS AND $\tilde{Q}_{P}\left(X_{B}\right)$ IN TERMS OF PROTON WAVE FUNCTION AMPLITUDES}

In this section we present explicit expressions needed to compute the quark PDFs and the twist-four distribution function.

The distribution functions $\tilde{Q}_{p}^{ \pm}\left(x_{B}\right)$ in Eqs. (29) and (30) are expressed in terms of $\psi_{l_{z}}^{ \pm}\left(q, l, q^{\prime}, l^{\prime}\right)$, which have the following expressions:

$$
\begin{aligned}
\psi_{l_{z}=0}^{+}\left(q, l, q^{\prime}, l^{\prime}\right)= & 2 \psi^{(1,2) *}(q, 1, l) \psi^{(1,2)}\left(q^{\prime}, 1, l^{\prime}\right) \\
\psi_{l_{z}=0}^{-}\left(q, l, q^{\prime}, l^{\prime}\right)= & 2\left\{\psi^{(1,2) *}(1, q, l) \psi^{(1,2)}\left(1, q^{\prime}, l^{\prime}\right)+\psi^{(1,2) *}(q, l, 1) \psi^{(1,2)}\left(q^{\prime}, l^{\prime}, 1\right)+\psi^{(1,2) *}(1, l, q) \psi^{(1,2)}\left(q^{\prime}, l^{\prime}, 1\right)\right. \\
& \left.+\psi^{(1,2) *}(q, l, 1) \psi^{(1,2)}\left(1, l^{\prime}, q^{\prime}\right)+\psi^{(1,2) *}(1, l, q) \psi^{(1,2)}\left(1, l^{\prime}, q^{\prime}\right)\right\} \\
\psi_{l_{z}=1}^{+}\left(q, l, q^{\prime}, l^{\prime}\right)= & 2 \psi^{(3,4) *}(1, q, l) \psi^{(3,4)}\left(1, q^{\prime}, l^{\prime}\right) \\
\psi_{l_{z}=1}^{-}\left(q, l, q^{\prime}, l^{\prime}\right)= & 2\left\{\psi^{(3,4) *}(q, 1, l) \psi^{(3,4)}\left(q^{\prime}, 1, l^{\prime}\right)+\psi^{(3,4) *}(l, q, 1) \psi^{(3,4)}\left(l^{\prime}, q^{\prime}, 1\right)+\psi^{(3,4) *}(l, 1, q) \psi^{(3,4)}\left(l^{\prime}, q^{\prime}, 1\right)\right. \\
& \left.+\psi^{(3,4) *}(l, q, 1) \psi^{(3,4)}\left(l^{\prime}, 1, q^{\prime}\right)+\psi^{(3,4) *}(l, 1, q) \psi^{(3,4)}\left(l^{\prime}, 1, q^{\prime}\right)\right\} \\
\psi_{l_{z}=-1}^{+}\left(q, l, q^{\prime}, l^{\prime}\right)= & 2\left\{\psi^{(5,5) *}(q, 1, l) \psi^{(5,5)}\left(q^{\prime}, 1, l^{\prime}\right)+\psi^{(5,5) *}(1, q, l) \psi^{(5,5)}\left(q^{\prime}, 1, l^{\prime}\right)\right. \\
& \left.+\psi^{(5,5) *}(q, 1, l) \psi^{(5,5)}\left(1, q^{\prime}, l^{\prime}\right)+\psi^{(5,5) *}(1, q, l) \psi^{(5,5)}\left(1, q^{\prime}, l^{\prime}\right)\right\}
\end{aligned}
$$




$$
\begin{aligned}
\psi_{l_{z}=-1}^{-}\left(q, l, q^{\prime}, l^{\prime}\right)= & 0 \\
\psi_{l_{z}=2}^{+}\left(q, l, q^{\prime}, l^{\prime}\right)= & 2\left\{\psi^{(6,6) *}(q, 1, l) \psi^{(6,6)}\left(q^{\prime}, 1, l^{\prime}\right)+\psi^{(6,6) *}(1, q, l) \psi^{(6,6)}\left(q^{\prime}, 1, l^{\prime}\right)\right. \\
& \left.+\psi^{(6,6) *}(q, 1, l) \psi^{(6,6)}\left(1, q^{\prime}, l^{\prime}\right)+\psi^{(6,6) *}(1, q, l) \psi^{(6,6)}\left(1, q^{\prime}, l^{\prime}\right)\right\} \\
\psi_{l_{z}=2}^{-}\left(q, l, q^{\prime}, l^{\prime}\right)= & 0 .
\end{aligned}
$$

The definitions of $\psi^{(i, j)}$ are the following:

$$
\begin{aligned}
& \psi^{(1,2)}(1,2,3)=\psi^{(1)}(1,2,3)+i\left(k_{1}^{x} k_{2}^{y}-k_{1}^{y} k_{2}^{x}\right) \psi^{(2)}(1,2,3) \\
& \psi^{(3,4)}(1,2,3)=k_{1 \perp}^{+} \psi^{(3)}(1,2,3)+k_{2 \perp}^{+} \psi^{(4)}(1,2,3) \\
& \psi^{(5,5)}(1,2,3)=-k_{2 \perp}^{-} \psi^{(5)}(1,2,3)+k_{3 \perp}^{-} \psi^{(5)}(1,3,2) \\
& \psi^{(6,6)}(1,2,3)=k_{1 \perp}^{+}\left(k_{2 \perp}^{+} \psi^{(6)}(1,3,2)-k_{3 \perp}^{+} \psi^{(6)}(1,2,3)\right) .
\end{aligned}
$$

On the other hand, the quark distribution functions in (27) are given in terms of $A^{l_{z}}(q, 1,2)$, which look like the following:

$$
\begin{aligned}
A^{l_{z}=0}(q, 1,2)= & \psi^{(1,2) *}(q, 1,2) \psi^{(1,2)}(q, 1,2)+2 \psi^{(1,2) *}(1, q, 2) \psi^{(1,2)}(1, q, 2)+\psi^{(1,2) *}(q, 2,1) \psi^{(1,2)}(q, 2,1) \\
& +\psi^{(1,2) *}(1,2, q) \psi^{(1,2)}(q, 2,1)+\psi^{(1,2) *}(q, 2,1) \psi^{(1,2)}(1,2, q)+2 \psi^{(1,2) *}(1,2, q) \psi^{(1,2)}(1,2, q) \\
& +\psi^{(1,2) *}(1, q, 2) \psi^{(1,2)}(2, q, 1)
\end{aligned}
$$

$$
\begin{aligned}
A^{l_{z}=1}(q, 1,2)= & 2 \psi^{(3,4) *}(q, 1,2) \psi^{(3,4)}(q, 1,2)+\psi^{(3,4) *}(1, q, 2) \psi^{(3,4)}(1, q, 2)+\psi^{(3,4) *}(2, q, 1) \psi^{(3,4)}(2, q, 1) \\
& +\psi^{(3,4) *}(2,1, q) \psi^{(3,4)}(2, q, 1)+\psi^{(3,4) *}(2, q, 1) \psi^{(3,4)}(2,1, q)+\psi^{(3,4) *}(2,1, q) \psi^{(3,4)}(2,1, q) \\
& +\psi^{(3,4) *}(1,2, q) \psi^{(3,4)}(1,2, q)+\psi^{(3,4) *}(q, 1,2) \psi^{(3,4)}(q, 2,1)
\end{aligned}
$$

$$
\begin{aligned}
A^{l_{z}=-1}(q, 1,2)= & \psi^{(5,5) *}(q, 1,2) \psi^{(5,5)}(q, 1,2)+\psi^{(5,5) *}(1, q, 2) \psi^{(5,5)}(q, 1,2)+\psi^{(5,5) *}(q, 1,2) \psi^{(5,5)}(1, q, 2) \\
& +\psi^{(5,5) *}(1, q, 2) \psi^{(5,5)}(1, q, 2)+\psi^{(5,5) *}(1,2, q) \psi^{(5,5)}(1,2, q)+\psi^{(5,5) *}(1,2, q) \psi^{(5,5)}(2,1, q)
\end{aligned}
$$

$$
A^{l_{z}=2}(q, 1,2)=\psi^{(6,6) *}(q, 1,2) \psi^{(6,6)}(q, 1,2)+\psi^{(6,6) *}(1, q, 2) \psi^{(6,6)}(q, 1,2)+\psi^{(6,6) *}(q, 1,2) \psi^{(6,6)}(1, q, 2)
$$$$
+\psi^{(6,6) *}(1, q, 2) \psi^{(6,6)}(1, q, 2)+\psi^{(6,6) *}(1,2, q) \psi^{(6,6)}(1,2, q)+\psi^{(6,6) *}(1,2, q) \psi^{(6,6)}(2,1, q)
$$

with $q=\left(x_{B} p^{+}, \vec{q}_{\perp}\right), x_{2}=1-x_{B}-x_{1}$ and $\vec{k}_{2 \perp}=-\vec{q}_{\perp}-\vec{k}_{1 \perp}$.

[1] C. Prescott et al., Phys. Lett. B 77, 347 (1978); 84, 524 (1979).

[2] P. Bosted et al., Jefferson-Lab Report No. PR-09-012, http://hallaweb.jlab.org/collab/PAC/PAC34/PR-09-012-pvdis. pdf (unpublished).

[3] M. R. Buckley and M. J. Ramsey-Musolf, Phys. Lett. B 712, 261 (2012).

[4] M. Gonzalez-Alonso and M. J. Ramsey-Musolf, Phys. Rev. D 87, 055013 (2013).

[5] A. Kurylov, M. J. Ramsey-Musolf, and S. Su, Phys. Lett. B 582, 222 (2004).

[6] See, e.g. R. L. Jaffe, Lectures on QCD: Applications (Springer, Berlin, 1997).

[7] P. Castorina and P. J. Mulders, Phys. Rev. D 31, 2760 (1985).

[8] S. Mantry, M. J. Ramsey-Musolf, and G. F. Sacco, Phys. Rev. C 82, 065205 (2010).

[9] A. V. Belitsky, A. Manashov, and A. Schäfer, Phys. Rev. D 84, 014010 (2011).

[10] T. Hobbs and W. Melnitchouk, Phys. Rev. D 77, 114023 (2008).

[11] J. Ashman et al., Nucl. Phys. B 328, 1 (1989).

[12] J. Ellis and R. L. Jaffe, Phys. Rev. D 9, 1444 (1974).

[13] M. Burkardt and H. BC, Phys. Rev. D 79, 071501 (2009).

[14] S. V. Bashinsky and R. L. Jaffe, Nucl. Phys. B 536, 303 (1998).

[15] X. Ji, X. Xiong, and F. Yuan, arXiv:1207.5221v1 [hep-ph].

[16] X. Ji, Phys. Rev. Lett. 78, 610 (1997).
[17] X. Ji, X. Xiong, and F. Yuan, Phys. Rev. Lett. 109, 152005 (2012).

[18] B. Pasquini and F. Yuan, Phys. Rev. D 81, 114013 (2010).

[19] B. Pasquini, S. Cazzaniga, and S. Boffi, Phys. Rev. D 78, 034025 (2008)

[20] R. N. Cahn and F. J. Gilman, Phys. Rev. D 17, 1313 (1978).

[21] M. Anselmino, M. Boglione, U. D’Alesio, S. Melis, F. Murgia, E. R. Nocera, and A. Prokudin, Phys. Rev. D 83, 114019 (2011).

[22] J. D. Bjorken, Phys. Rev. D 18, 3239 (1978).

[23] L. Wolfenstein, Nucl. Phys. B 146, 477 (1978).

[24] I. I. Balitsky and V. M. Braun, Nucl. Phys. B 311, 541 (1989).

[25] X. Ji, J. P. Ma, and F. Yuan, Nucl. Phys. B 652, 383 (2003).

[26] F. Schlumpf, Phys. Rev. D 47, 4114 (1993).

[27] S. Boffi, B. Pasquini, and M. Traini, Nucl. Phys. B 649, 243 (2003).

[28] A. Anselmino et al., arXiv:1107.4446v2 [hep-ph].

[29] A. Accardi, M. E. Christy, C. E. Keppel, W. Melnitchouk, P. Monaghan, J. G. Morfin, and J. F. Owens, Phys. Rev. D 81, 034016 (2010).

[30] W. B. Wood and G. B. West, Phys. Rev. D 7, 773 (1973).

[31] T. Hahn, Comput. Phys. Commun. 168, 78 (2005).

[32] S. Bethke, Eur. Phys. J. C 64, 689 (2009).

[33] D. W. Sivers, Phys. Rev. D 41, 83 (1990); 43, 261 (1991).

[34] D. Boer and P. J. Mulders, Phys. Rev. D 57, 5780 (1998).

[35] X. Ji, J. P. Ma, and F. Yuan, Eur. Phys. J 33, 75 (2004). 